\title{
Nonlinear Heart Rate Variability Measures During the Oral Glucose Tolerance Test
}

\author{
Gilberto Perpiñan ${ }^{1,3}$, Erika Severeyn ${ }^{1}$, Sara Wong $^{2}$, Miguel Altuve ${ }^{3}$ \\ ${ }^{1}$ Applied Biophysics and Bioengineering Group, Simon Bolivar University, Caracas, Venezuela \\ ${ }^{2}$ Department of Electrical, Electronics and Telecommunications Engineering, University of Cuenca, \\ Ecuador \\ ${ }^{3}$ Faculty of Electrical and Electronic Engineering, Pontifical Bolivarian University, Bucaramanga, \\ Colombia
}

\begin{abstract}
Heart rate variability (HRV) exhibits a different response to different external stimuli (emotional, auditory, etc). In this paper, HRV irregularity and correlations in short time scales were assessed after a sudden increase in plasma glucose concentration (a stimulus of glucose), using approximate entropy (ApEn) and short-term fluctuation ( $\alpha 1)$ of detrended fluctuation analysis, respectively, in people with metabolic syndrome and healthy control subjects. 5-min RR interval time series were extracted from ECG recordings acquired at five timed intervals $(0,30, \ldots, 120 \mathrm{~min})$ of an oral glucose tolerance test. We have found that, in fasting and $30 \mathrm{~min}$ after the glucose stimulus, people with metabolic syndrome showed statistically significantly greater values of ApEn than control, an indicative of a more irregular HRV. In addition, $60 \mathrm{~min}$ after the glucose stimulus, when plasma glucose concentration reaches its maximum value, HRV irregularity and short-term HRV correlation showed a reduction in people with metabolic syndrome. Further studies, using other nonlinear measures and a larger dataset, must be carried to characterize the response of HRV to the glucose stimulus.
\end{abstract}

\section{Introduction}

One of the major and escalating clinical and public health problem worldwide is the metabolic syndrome, a collection of cardiometabolic disorders (abdominal obesity, fasting hyperglycemia, hypertension, hypertriglyceridemia and decreased blood high-density lipoprotein (HDL) cholesterol) that increase the risk for cardiovascular disease, type 2 diabetes and stroke. A common screening tool to check for glucose tolerance and diabetes is the oral glucose tolerance test (OGTT), in which fasting plasma glucose concentration is measured, then, after oral glucose loading, plasma glucose concentration is measured again each 30 or $60 \mathrm{~min}$ for two to three hours. In the OGTT, the ingested dose of glucose can be seen as an external stimulus that could influence short-term and long-term heart rate dynamics. Indeed, it has been shown that heart rate variability (HRV) is affected, in different ways, by a variety of stimuli (emotional, auditory, ... ) $[1,2]$. This variability is nothing more than the result of the modulation exerted in the heart by the sympathetic and parasympathetic components of the autonomic nervous system.

The assessment of HRV is commonly performed using time-domain and frequency-domain measures; however, the nonlinear nature of HRV, as the vast majority of biological signals, has led to the use of chaos and complexity of fractal dynamics techniques to assess cardiac autonomic modulation and extract clinical information that are not observable using traditional linear methods. In this sense, Lyapunov exponents, Poincaré plot, approximate entropy (ApEn), correlation dimension and detrended fluctuation analysis (DFA) are useful techniques to assess HRV from a nonlinear point of view.

Healthy systems show higher values of ApEn, which translates into greater complexity or irregularity, than pathological ones. For instance, patients with type 2 diabetes show lower complexity of HRV (lower values of ApEn) than healthy subjects [3], however, patients with heart rate failure show higher complexity of HRV (higher values of ApEn) than healthy subjects [4]. On the other hand, short-term fractal scaling exponent $\alpha 1$ of DFA has been used to understand the regulatory mechanisms of the autonomic nervous system and the randomness of HRV. Fetal HRV fractal correlations $(\alpha 1)$ and irregularity (ApEn) have been found to be increased after vibro-acustic stimulation of the mother's abdomen [2].

In this work we use ApEn and $\alpha 1$ of DFA measures to investigate whether a sudden increase in plasma glucose concentration (a stimulus of glucose) can modify shortterm HRV irregularity and fractal correlations, respectively. 
This hypothesis is evaluated using a dataset of people diagnosed with metabolic syndrome and age-matched control subjects during an OGTT.

\section{Materials and methods}

\subsection{Dataset}

The dataset is composed of two groups: fifteen male subjects diagnosed with the metabolic syndrome according to the National Cholesterol Education Program-Adult Treatment Panel (NCEP ATP III) [5], and ten male, agematched, control subjects. 12-lead ECG recordings and plasma glucose and insulin concentrations were registered in fasting and at each $30 \mathrm{~min}$, after oral intake of $75 \mathrm{gr}$ of glucose, for two hours. ECG signals were recorded in seated position during $10 \mathrm{~min}$ approximately at each timed intervals $(0,30, \ldots, 120 \mathrm{~min})[6,7]$. Five of the control subjects were removed from the dataset due to the presence of at least, one of the metabolic syndrome factor. Table 1 shows the median value (interquartile range, IQR) of the measured variables, for each study group.

\subsection{Nonlinear analysis of $\mathrm{HRV}$}

The beat-to-beat interval variability or HRV was assessed through the analysis of short-term RR interval time series extracted from ECG recordings, where R corresponds to the peak of the QRS complex and RR corresponds to the successive differences of two R peaks. For short-term HRV analysis (5 min), RR interval time series were extracted from minutes 4 to 9 of the ECG recordings. Accurate R-peak detection was performed by combining individual R-peak detections made on each ECG lead using a data fusion technique [8].

Kubios software was used for HRV analysis [9]. RR intervals differing from the previous one by more than $30 \%$ were identified as artifacts and were corrected using $\mathrm{Ku}-$ bios' custom filter. To explore HRV irregularity and shortterm correlations on different time scales, two nonlinear HRV parameters, ApEn and $\alpha_{1}$ from DFA, were obtained using Kubios.

ApEn quantifies the irregularity of the RR interval series and measures the disorder in the heart rate signal. Mathematically speaking, ApEn corresponds to the difference between the logarithmic frequencies of similar segments of length $m$ and segments with length $m+1$. ApEn of a time series measures the logarithmic likelihood that patterns of length $m$ that are close to each other in RR interval series will remain close in the next incremental comparisons, $m+1$. In our experiments, $m$ was set to 2 .

DFA measures the correlation within the RR interval time series over different time scales $[10,11]$. For each $k=1, \ldots, N$, the RR interval time series $(N \approx 300$ beats $)$ is mapped to $y(k)$, according to equation 1 , where $\overline{R R}$ is the average of the $R R_{j}$ interval time series.

$$
y(k)=\sum_{j=1}^{k}\left(R R_{j}-\overline{R R}\right), k=1, \ldots, N .
$$

$y(k)$ is then divided into segments of equal length $n$ to obtain $y_{n}(k)$, the local trend within each segment, which is the least squares line fitted to the segment. $F$, as a function of the length $n$, is computed for different segment lengths, according to equation 2. Short-term correlations of HRV are represented by $\alpha_{1}$, the slope of the regression line of $\log F(n)$ as function of $\log n$, in the range $4 \leq n \leq 16$ heartbeats. Figure 1 shows an example of $\alpha_{1}$ for a subject with metabolic syndrome and a control subject.

$$
F(n)=\sqrt{\frac{1}{N} \sum_{k=1}^{N}\left(y(k)-y_{n}(k)\right)^{2}}
$$

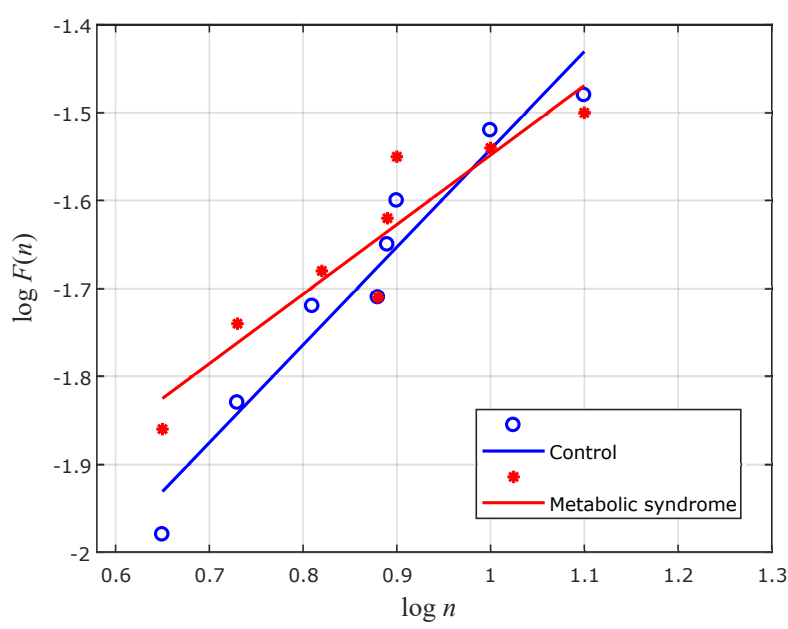

Figure 1: Example of short-term correlations of HRV for a subject with metabolic syndrome $(\alpha 1=1.07)$ and a control subject $(\alpha 1=1.04)$ from our dataset.

\subsection{Statistical analysis}

Statistically significant differences in the median of the parameters, ApEn and $\alpha_{1}$, between groups (metabolic syndrome and control) were assessed using the two-sided nonparametric Wilcoxon rank-sum test, since these are nonparametric, non-normal independent samples. In addition, statistically significant differences among the timed intervals of the OGTT for each group were assessed using the two-sided non-parametric Friedman test and Tukey's honest post hoc analysis, since these are dependent, nonparametric samples. A $p$-value less than 0.05 was considered statistically significant. 
Table 1: Median (IQR) of the measured attributes for each study group. $p$-values were obtained from the Wilcoxon ranksum test for nonparametric and not normal distribution data.

\begin{tabular}{llll}
\hline Attribute & Metabolic syndrome & Control & $p$-value \\
\hline Age $($ year) & $33(8)$ & $29(9)$ & 0.11 \\
Waist Circumference $(\mathrm{cm})$ & $108.8(18.0)$ & $87.5(16.6)$ & 0.027 \\
Fasting plasma glucose $(\mathrm{mg} / \mathrm{dL})$ & $109(8.5)$ & $95(4)$ & $<0.001$ \\
Fasting plasma insulin $(\mu \mathrm{UL} / \mathrm{mL})$ & $11(6.5)$ & $2(3)$ & 0.018 \\
Blood pressure $(\mathrm{mmHg})$ & $137(6.5) / 88(17.8)$ & $116(7.5) / 76(17)$ & $<0.01 / 0.03$ \\
Triglycerides $(\mathrm{mg} / \mathrm{dL})$ & $195(135.8)$ & $72(33.5)$ & $<0.001$ \\
HDL cholesterol $(\mathrm{mg} / \mathrm{dL})$ & $41(10.5)$ & $47.0(10.0)$ & 0.19 \\
\hline
\end{tabular}

\section{Results}

Table 2 shows the median (IQR) of the nonlinear HRV measures, ApEn and $\alpha_{1}$, for each stage of the test. In addition, Figures 2 and 3 show ApEn and $\alpha 1$, respectively, during the test for subjects with metabolic syndrome (Mets) and control (Ctrl).

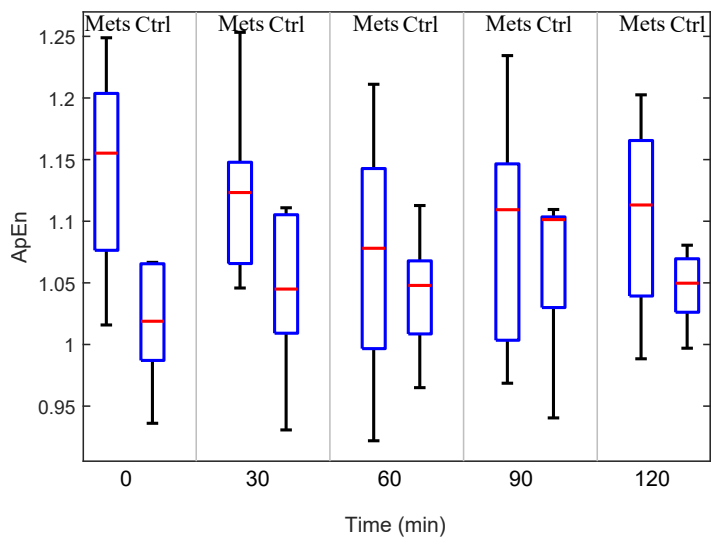

Figure 2: Box plot of ApEn during the OGTT. Mets stands for metabolic syndrome and Ctrl stands for control.

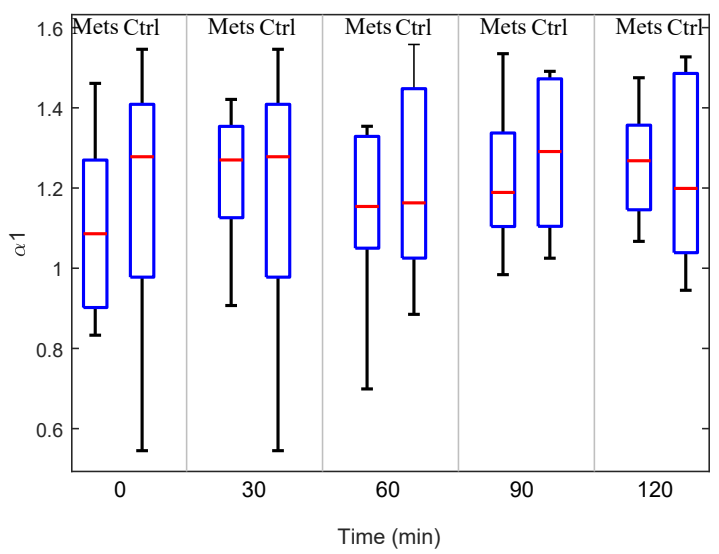

Figure 3: Box plot of $\alpha 1$ during the OGTT. Mets stands for metabolic syndrome and Ctrl stands for control.
We have observed that ApEn was statistically significantly greater, i.e. more irregular, in subjects with metabolic syndrome compared to control people in fasting (1.16 vs. $1.04, p=0.018$ ) and $30 \mathrm{~min}$ after the intake of glucose (1.12 vs. 1.06, $p=0.008)$. For $\alpha_{1}$, we have not found statistically significant differences between groups for the timed intervals.

Concerning the differences between the timed intervals of the OGTT, we have not found statistically significant differences neither for ApEn nor for $\alpha 1$ for each group.

\section{Discussion}

We have found that subject with metabolic syndrome, after prolonged fast and $30 \mathrm{~min}$ after the intake of glucose, showed statistically significantly greater irregularity in the HRV than control people. It would be expected that subjects with metabolic syndrome would have shown less irregularity than control people because hypertension, a condition of the metabolic syndrome, cause a reduction in the HRV irregularity [12]. However, a similar result has been reported in the literature for ApEn, in which people with heart failure show higher values of ApEn compared to healthy people, an indicative of more erratic heart rate pattern for a pathological system [4].

After the glucose stimulus, HRV irregularity decreases in subjects with metabolic syndrome, especially at $60 \mathrm{~min}$, where plasma glucose concentration reach its higher values. This behavior is not observed in the control population. Even if the reduction of HRV irregularity in subjects with metabolic syndrome among the stages of the OGTT is not statistically significant, it could be a reflex of the excess of glucose in blood and the inefficacy of the system to cope with it. Similarly, the reduction of the short-scaling correlation of HRV observed in subjects with metabolic syndrome at the same timed interval (min 60) might be a lagged response of the autonomic nervous system to cope with the increased plasma glucose concentration. 
Table 2: Median (IQR) of ApEn and $\alpha_{1}$ during the OGTT.

\begin{tabular}{lllllll}
\hline Variable & Group & $0 \mathrm{~min}$ & $30 \mathrm{~min}$ & $60 \mathrm{~min}$ & $90 \mathrm{~min}$ & $120 \mathrm{~min}$ \\
\hline \multirow{2}{*}{ ApEn } & Metabolic syndrome & $1.16(0.13) \dagger$ & $1.12(0.08) \ddagger$ & $1.08(0.15)$ & $1.11(0.14)$ & $1.11(0.13)$ \\
& Control & $1.02(0.08)$ & $1.04(0.10)$ & $1.05(0.06)$ & $1.10(0.07)$ & $1.05(0.04)$ \\
\hline \multirow{2}{*}{$\alpha_{1}$} & Metabolic syndrome & $1.09(0.37)$ & $1.27(0.23)$ & $1.15(0.28)$ & $1.19(0.23)$ & $1.27(0.21)$ \\
& Control & $1.28(0.43)$ & $1.17(0.29)$ & $1.16(0.42)$ & $1.29(0.37)$ & $1.20(0.45)$ \\
\hline
\end{tabular}

$\dagger$ Statistically significantly greater than in control group.

‡ Statistically significantly greater than in control group.

\section{Conclusion}

This study has shown that a sudden increase of plasma glucose concentration in subjects with metabolic syndrome may be observed in the cardiovascular system as a reduction in the irregularity of the HRV. This, in fact, could be seen as a reflected lagged response in the cardiac autonomic modulation to handle with the increased plasma glucose concentration (a metabolic response). However, further studies must be carried out using a larger dataset. In addition, the assessment of the HRV response to the stimulus of glucose should be carried out using other nonlinear measures, like multiscale entropy or LempelZiv complexity, in order to find clinically relevant indicators to diagnose the metabolic syndrome and related diseases and enhance our understanding of the mechanism involved in cardiac autonomic modulation.

\section{Acknowledgments}

This work was partially supported by the Office of Research and Transfer of Pontifical Bolivarian University, and by the Research Direction of the University of Cuenca, Ecuador (DIUC-Dirección de Investigación de la Universidad de Cuenca, Ecuador).

\section{References}

[1] Fujimura T, Okanoya K. Heart rate variability predicts emotional flexibility in response to positive stimuli. Psychology 2012;3(08):578.

[2] Ferrario M, Signorini M, Magenes G. The fetal heart rate variability due to vibro-acustic stimulation: a complexity analysis. In 4th European Conference of the International Federation for Medical and Biological Engineering. Springer, 2009; 1353-1356.

[3] Li X, Yu S, Chen H, Lu C, Zhang K, Li F. Cardiovascular autonomic function analysis using approximate entropy from 24-h heart rate variability and its frequency components in patients with type 2 diabetes. Journal of diabetes investigation 2015;6(2):227-235.

[4] Beckers F, Ramaekers D, Aubert AE. Approximate entropy of heart rate variability: validation of methods and appli- cation in heart failure. Cardiovascular Engineering 2001; 1(4):177-182.

[5] Grundy SM, Brewer HB, Cleeman JI, Smith SC, Lenfant $\mathrm{C}$, et al. Definition of metabolic syndrome report of the National Heart, Lung, and Blood Institute/American Heart Association Conference on scientific issues related to definition. Circulation 2004;109(3):433-438.

[6] Severeyn E, Wong S, Passariello G, Cevallos JL, Almeida D. Methodology for the study of metabolic syndrome by heart rate variability and insulin sensitivity. Revista Brasileira de Engenharia Biomedica 2012;28(3):272-277.

[7] Ledezma CA, Severeyn E, Perpinan G, Altuve M, Wong S. A new on-line electrocardiographic records database and computer routines for data analysis. In Engineering in Medicine and Biology Society (EMBC), 2014 36th Annual International Conference of the IEEE. 2014; 2738-2741.

[8] Ledezma CA, Perpiñan G, Severeyn E, Altuve M. Data fusion for qrs complex detection in multi-lead electrocardiogram recordings. In 11th International Symposium on Medical Information Processing and Analysis (SIPAIM 2015). International Society for Optics and Photonics, 2015; 968118-968118.

[9] Tarvainen MP, Niskanen JP, Lipponen JA, Ranta-Aho PO, Karjalainen PA. Kubios hrv-heart rate variability analysis software. Computer methods and programs in biomedicine 2014;113(1):210-220.

[10] Acharya R, Lim C, Joseph P. Heart rate variability analysis using correlation dimension and detrended fluctuation analysis. ITBM RBM 2002;23(6):333-339.

[11] Peng CK, Havlin S, Stanley HE, Goldberger AL. Quantification of scaling exponents and crossover phenomena in nonstationary heartbeat time series. Chaos An Interdisciplinary Journal of Nonlinear Science 1995;5(1):82-87.

[12] Virtanen R, Jula A, Kuusela T, Helenius H, Voipio-Pulkki LM. Reduced heart rate variability in hypertension: associations with lifestyle factors and plasma renin activity. Journal of human hypertension 2003;17(3):171.

Address for correspondence:

Miguel Altuve

Km. 7 Autopista a Piedecuesta, Universidad Pontificia Bolivariana, Bucaramanga 681008, Colombia.

miguel.altuve@upb.edu.co 